\title{
Determining Forces of Self-Assembly in Thin Films by measuring Thermal Vibrations.
}

Konrad Thürmer

Sandia National Laboratories, P.O. Box 969, Livermore, California 94551

Self-assembly of surfaces into stable periodic patterns requires the presence of stabilizing forces between neighboring surface features. Controlling these stabilizing forces holds the key of tailoring self-assembled structures, provided kinetic limitations can be overcome by a high surface mobility. In this work we analyze the thermal vibrations in the periodic pattern of a well-defined model system, $\mathrm{S} / \mathrm{Ag} / \mathrm{Ru}$, with the goal of determining the nature of forces responsible for self-assembly. We find that these forces originate from interactions between misfit dislocations in the Ag film.

Deposition of sulfur onto a monolayer of $\mathrm{Ag}$ on $\mathrm{Ru}(0001)$ transforms the clean Ag-film into a strikingly regular array of 2D-vacancy islands [1]. Our real-time STM measurements allow us to probe the formation and mechanical stability of this self-assembled structure. We have shown that this nanometer-scale restructuring proceeds via the rearrangement of misfit dislocations. In STM image sequences of the final structure we observe that the lattice of S-filled vacancy islands vibrates. We have analyzed these thermal vibrations to determine the forces, which stabilize the structure. Fig.1 (a) visualizes the motion of the S-filled holes in an overlay of two subsequently acquired STM images. The strong temperature dependence of these vibrations is evidence of their thermal origin. The time-correlation function $\mathrm{G}(\tau)$ of the separations of the nearest-neighbor $(\mathrm{NN})$ holes is a good measure for the time-scale on which the vacancy islands move. From an experimental plot of $G(\tau)$ (Fig1b) we conclude that at room temperature the vibrations are slow enough to be captured by STM. This allows a detailed analysis of the excursions of the vacancy islands from their equilibrium positions in the triangular lattice. Fig. 1(c) shows a histogram of the fluctuations of the NN-hole separation at room temperature. The width of this almost perfect Gaussian distribution contains information about the restoring force. An analysis of the anisotropy of these thermal vibrations elucidates the nature of these forces. The measured vibrational component along the connecting line of a NN-hole pair (stretch mode) is slightly larger than the perpendicular "shear" component (Fig.1e), whereas the simple spring model predicts a stretch component only 0.7 times that of the shear component. Our measurements thus reveal a significant force component perpendicular to the connecting line. A 2D Frenkel-Kontorova model (Fig.2) naturally explains the observed hole-hole interaction anisotropy as a property of the dislocation network in the Ag film.

We found a mechanism for self-assembly based on the properties of a network of misfit dislocations. Controlling the misfit through varying the composition of the film, in this case a $\mathrm{Ag}_{\mathrm{x}} \mathrm{Cu}_{1-\mathrm{x}}$ alloy, holds the promise of tailoring the spacing in self-assembled lattices- a method potentially applicable for other misfit dislocation based self-assemblies.

[1] K. Pohl et al., Nature 397 (1999) 238.

[2] K. Thürmer, C.B. Carter, N.C. Bartelt, R.Q. Hwang, Phys. Rev. Lett. 92, 106101-1 (2004).

[3] Sandia is a multiprogram laboratory operated by Sandia Corporation, a Lockheed Martin Company, for the United States Department of Energy's National Nuclear Security Administration under contract DE-AC04-94AL85000. 
(a)

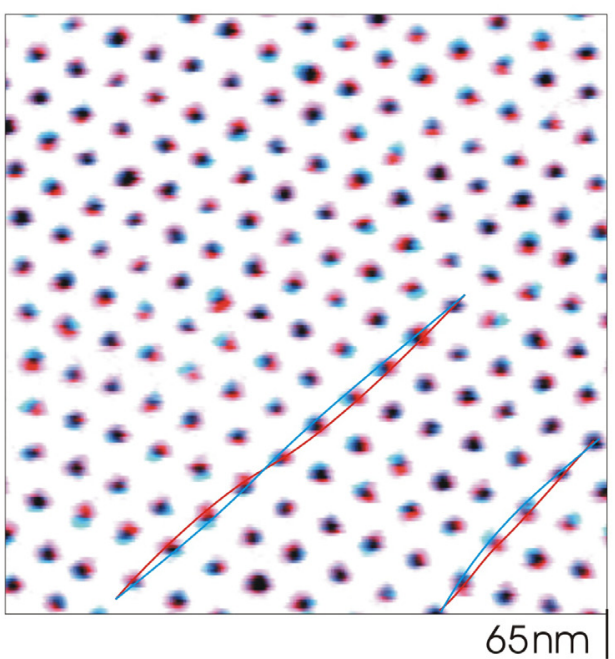

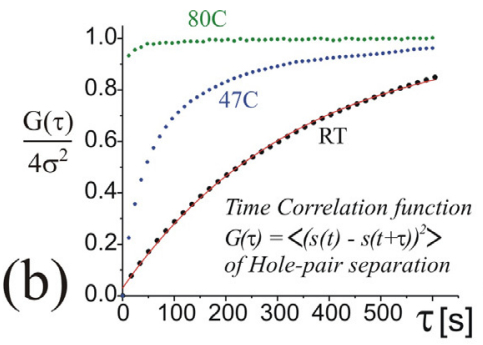

(c)



(d)

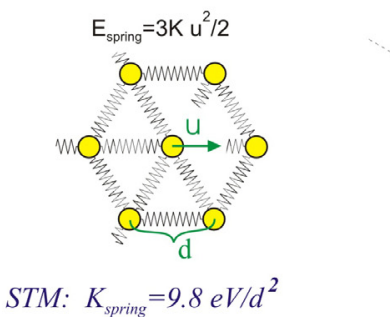

(e)

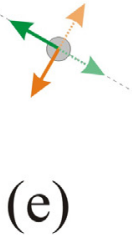

$<\mathrm{S}^{2}>/ \mathrm{d}^{2}=$

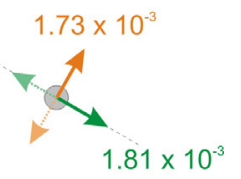

Fig.1. Measuring vibrations in a lattice of vacancy islands: (a) Superposition of two STM-images (red and cyan) acquired $12 \mathrm{~s}$ apart at $\mathrm{T}=80^{\circ} \mathrm{C}$. The red and the blue lines point to the presence of longer-wavelength vibrations in the lattice. (b) Time correlation function of the nearest neighbor (NN) hole pair separation. Its strong temperature dependence is evidence for the thermal origin of the vibrations. (c) The histogram of the fluctuations of NN-hole separations allows quantifying the restoring forces holding each vacancy in place. (d) Spring model of NN-hole interaction. (e) In contrast to predictions for the spring model we measured the vibrational component along the connecting line of a NN-hole pair (stretch mode) to be larger than the shear component.



(a)

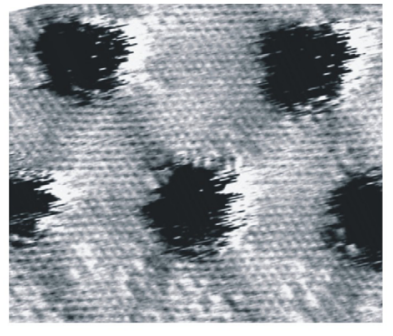

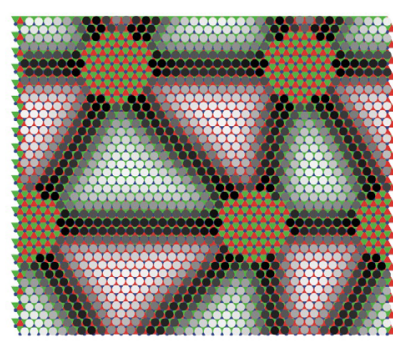

(b)

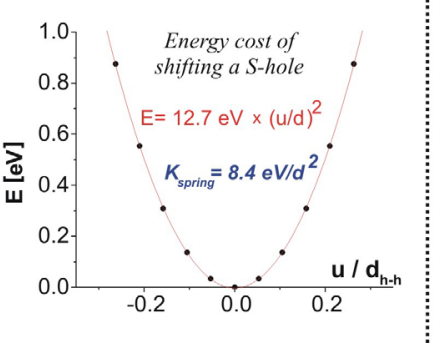

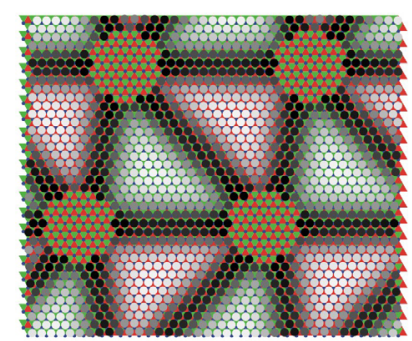

(c)

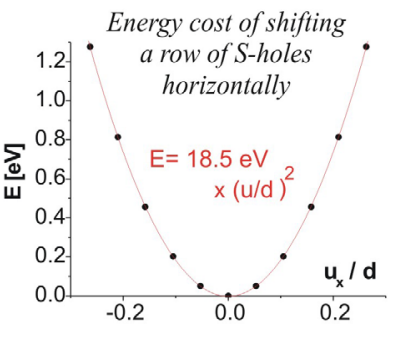

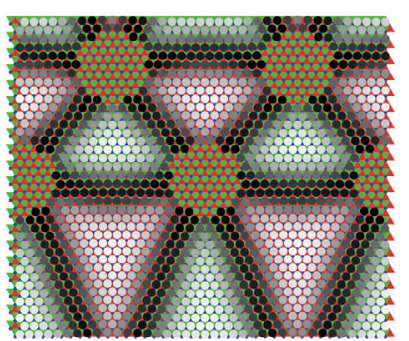

(d)

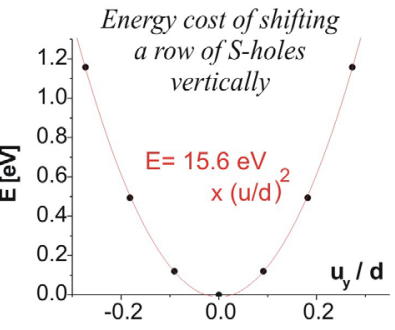

Fig.2. Probing the restoring forces in the vacancy lattice by means of a 2D-Frenkel-Kontorova model. (a) Top: unit cell used in the simulation. bottom: corresponding STM-image (b-d): Simulating the shift of one hole (b) or a row of holes (c, d) out of their equilibrium positions. Knowing the energy cost of these excitations allows us to determine the magnitude and the direction of the restoring forces. These Frenkel-Kontorova simulations let us conclude that the forces responsible for self-assembly in the $\mathrm{S} / \mathrm{Ag} / \mathrm{Ru}$-system originate in the misfit-dislocation network. 